\title{
$1 N-76$
}

NASA Technical Memorandum 105184

AIAA-91-3592

\section{8}

p. 8

\section{High Temperature Superconductor Analog Electronics for Millimeter- Wavelength Communications}

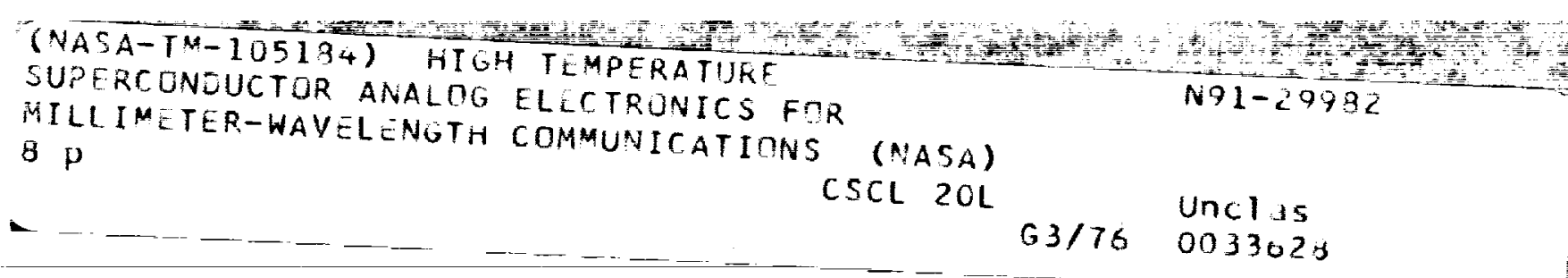

R.R. Romanofsky and K.B. Bhasin

Lewis Research Center

Cleveland, Ohio

Prepared for the

Conference on Advanced Space Exploration Initiative Technologies cosponsored by the AIAA, NASA, and OAI

Cleveland, Ohio, September 4-6, 1991 



\title{
HIOR TEMPERATURE BUPERCONDUCTOR ANALOG ELECTRONICB FOR MILLIMETER-TAVELENGTH CONGUNICATIONB
}

\author{
R. R. Romanofsky and K. B. Bhasin \\ NASA Lewis Research Center \\ 21000 Brookpark Road \\ cleveland, ohio $\mathbf{4 4 1 3 5}$
}

\section{ABSTRACY}

The performance of high-temperature superconductor (HTS) passive microwave circuits up to $X$-band has been encouraging when compared to their metalilc counterparts, The extremely low surface resistance of HTS films up to about $10 \mathrm{GHz}$ enables a reduction in loss by as much as 100 times compared to copper when both materials are kept at about 77K. However, a superconductor's surface resistance varies in proportion to the frequency squared. Consequently, the potential benefit of HTS materials to millimeter-wavs communications requires careful analysis. A simple ring resonator has been used to evaluate microstrip losses at Ka-band. We have also investigated additional promising components such as antennas and phase shifters. Prospects for HTs to favorably impact milimeter-wave communications systems are discussed.

\section{INTRODOCTION}

Thin film analog signal processing electronics was envisioned as the first practical application for high temperature superconductors. The major prerequisite for microwave circuits was comparatively low surface resistance as opposed to high current density or high critical magnetic field performance, although the parameters are correlated. The surface resistance (Rs) of normal conductors varies in proportion to the square root of frequency due to the skin effect, whereas for a superconductor the surface resistance is proportional to the frequency squared. The strong dependence of Rs on frequency for superconductors is a consequence of the reactive voltage assoclated with the oscillating supercurrent interacting with unpaired (normal) electrons to Induce loss. The proportion of normal electrons varles from 0 at $T=0$ to 1 at $T=T C$.

This property can be exploited to enhance passive microstrip circuitry, such as filters and phased array beam forming networks (BFNs). HTS microstrip enables tremendous miniaturization of filters without any concomitant degradation in performance compared to a waveguide implementation. HTS microstrip may also enable high directivity arrays because of the greatly reduced gain degradation in BFNs. Antennas, phase shifters, hybrid superconductor/semiconductor circuits and other components are likely to profit from this property as well. This paper will examine the potential of passive HTS circuitry to favorably impact millimeterwave communications.

The greatest contribution to millimeterwave communications may ultimately result from active low- or high-Tc superconducting electronics. Digital signal processors utilizing Josephson junction (JJ) technology will almost surely evolve because of the three-to-four orders of magnitude improvement in the delay-power product. LOW-TC JJs operate ten times faster than any high density semiconductor device and consume 1000 times less power. The eventual possibility of high-TC JJ chips has been suggested as researchers learn to intentionally manufacture arrays of large angle grain boundary junctions. It has been established for conventional superconductors that energy gap is approximately $3.5 \mathrm{kTC}$ at zero temperature where $k$ is Boltzmann's constant. This gap varies slowly with temperature up to about $1 / 2$ Tc. Beyond about $2 / 3$ Tc, the gap diminishes precipitously, becoming zero at $T=T C$. Consequently, in practice, device operation may be restricted to temperatures below 0.67 Tc. It cannot be stated with certainty that this same phenomenon will govern high-Tc electronics. A novel active high-Tc device termed the superconducting llux flow transistor (SFFT), may lead to a new class of microwave electronics. The sFFT uses a current control line to create a magnetic field and induce flux motion which changes the terminal voltage. Hence, the SFFT is the electrical dual of the FET. The SFFT can produce a net gain and has been demonstrated in basic microwave devices. Although this paper will not locus on active high-Tc electronics, revolutionary advances in communications and high speed data processing will likely be derived from this facet of the technology.

\section{Wicrostrip Rting Resonator}

Microstrip ring resonators with a design center frequency of $35 \mathrm{GHz}$ were fabricated from laser ablated YBacuo thin films 
deposited on lanthanum aluminate substrates ${ }^{2}$. The ring resonator technique provides a useful method for evaluating patterned $\mathrm{f} i \mathrm{~m}$ characteristics and includes film-substrate interface effects. Reflection coefficients were measured over a wide temperature range and performance was compared to identical resonators made of evaporated gold. The performance crossover, in terms of resonator " $Q$ " which is inversely proportional to loss, occurred at about $80 \mathrm{~K}$. The unloaded $Q$ of the superconducting resonator was about 4 times better than gold at $20 \mathrm{~K}$. Unloaded $Q$ data as a function of temperature is shown in figure 1. As expected because of the dependence of internal inductance of the strip on the changing normal/superconducting electron densities, the resonant frequency was observed to have a negative temperature coefficient. This prototype device indicated the potential for HTS to enhance millimeter-wave microstrip circuitry.

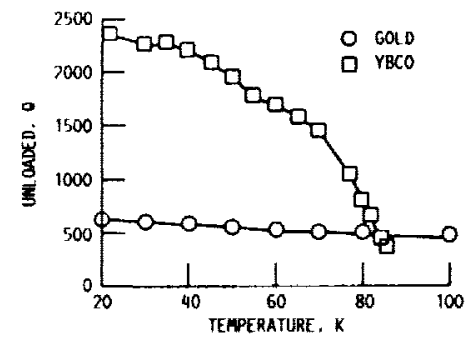

Figure 1 - 35 GHz RING RESONATOR UNLOAD "O" VERSUS TEMPERATURE FOR GOLD AND LASER ABLATED $\mathrm{YBa}_{2} \mathrm{Cu}, \mathrm{O}, \&$ FILM ON LAAIO

\section{Low Nolge Recelvers}

Because of the extraordinary distances involved and scarce transmitter power, receivers for deep-space links have extremely demanding sensitivity requirements. At the present time, maser amplifiers provide the only practical technology for interplanetary communications. The drawback to these systems is cost and the complexity of the necessary $4.5 \mathrm{~K}$ refrigeration. A promising alternative to maser technology is cryogenically cooled high electron mobility transistor (HEMT) low noise amplifiers (LNAS). Nolse figures of HEMTs cooled below liquid nitrogen temperatures are approaching the performance of maser amplifiers. The minimum noise figure of a metal semiconductor field effect transistor (MESFET) scales as the reciprocal of the cutoff frequency (or transconductance). The high carrier mobility of a HEMT results. in a much larger transconductance when compared to a conventional MESFET. Furthermore, the noise conductance is inversely proportional to the square of the cutoff frequency. This reduces the HEMT's susceptibility to impedance variations and enables high performance over a wide bandwidth. The HEMT's performance also improves much more rapidly upon cooling than a conventional MESFET.

A possible Mars-Earth communications scenario calls for relay satellites in orbit about each planet. Because of the increased operating temperature, reduced cost, and improved reliability, HEMT LNA receivers may prove enabling. In order to assess the performance enhancement that ITS could provide to such a system, mock hypothetical recelvers were designed at 10 , 32,60 , and $94 \mathrm{GHz}$. Each simplified receiver consisted of an ideal antenna, 1.5 wavelengths of feed loss, a five-pole bandpass filter, three ( 10 and $32 \mathrm{GHz})$, four (60 $\mathrm{GHz})$, or five $(90 \mathrm{GHz})$ gain stages, and a mixer. Scattering and noise parameters of the hypothetical HEMTS were consistęnt with state-of-the-art performance 3,4 . since the LNA gain was nearly $40 \mathrm{~dB}$ in each case, any marginal improvement in HEMT gain upon cooling was neglected since it would not impact system sensitivity. A surface resistance of $5 \mathrm{milliohms}$ at $30 \mathrm{GHz}$ with a frequency squared dependence was assumed for the HTS. Figure 2 shows the modeled recelver noise figure as a function of frequency for gold microstrip at $300 \mathrm{~K}$ and $77 \mathrm{~K}$ and for HTS at about 77K. The shapes of the curves are largely an artifact of the chosen device parameters and the curve fitting algorithm. Obvious advantages of HTS over gold are indicated at lower microwave frequencies. An improvement in sensitivity of about $0.1 \mathrm{~dB}$ exists at 60 GHz. The enhancement diminishes to zero at 80-90 $\mathrm{GHz}$.

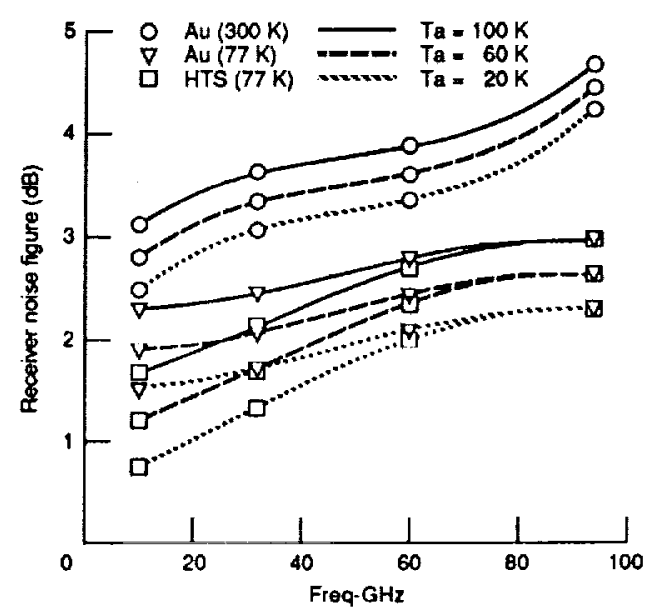

FIguTo 2 - PREDICIED RECEIVER SENSITIVITY ENHANCEMENT PROVIDEO BY PASSIVE HTS MICROSTRIP CIRCUITRY

The salient issue is the significance of the marginal improvements even at 
millimeter wavelengths. Figure 3 demonstrates the merit of even minor sensitivity improvements for a Mars-Earth link. Channel assumptions were based primarlly on an earlier study. For example, power efficient modulation permitting a receiver (energy per $\mathrm{bit}) /$ (noise power) ratio of $1.5 \mathrm{~dB}$ was assumed. A different range of $2.7 \times 10^{8}$ $\mathrm{km}$, which would place the planets roughly at quadrature, was assumed. Data is plotted for both five and ten meter transmit and receive antennas. A reduction in recelver noise figure by several tenths of a dB corresponds to a transmitter power reduction of tens to perhaps hundreds of watts, or alternatively to a reduction in antenna size. The development of large deployable antennas represents a significant technical challenge. Voyager, for example, utilized a 3.7 meter $X$-band antenna. The NASA Advanced Communication Technology Satellite's 3.2 meter dish is the largest Ka-band antenna developed to date.

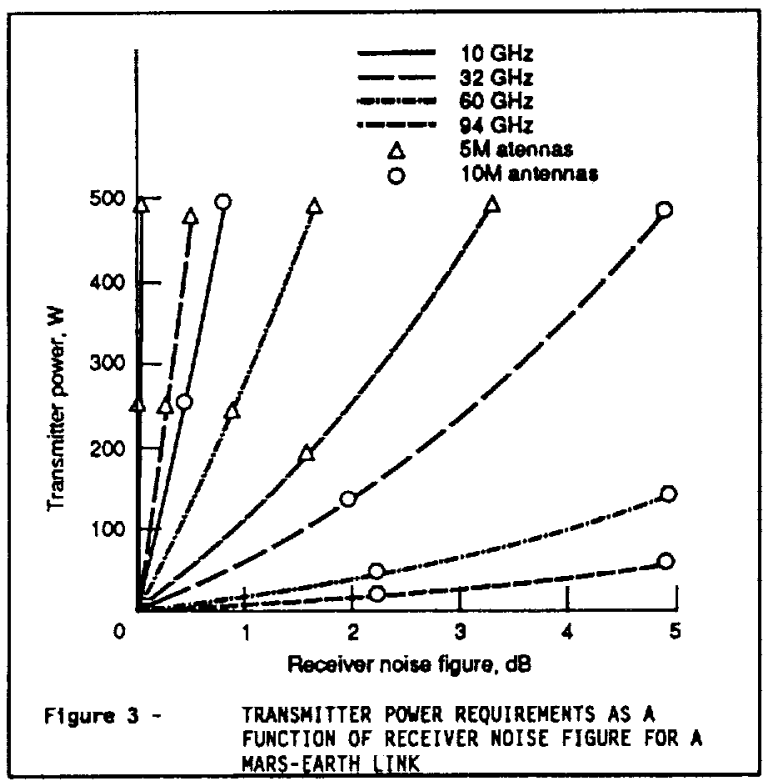

\section{superconduoting Miorestrip antennne}

Application of high Tc superconductivity to electrically short antennas, superdirective antenna arrays and millimeter wave array leed networks has been gnalyzed by several researchere recently 6,7 . Thelr work has shown HTS may be most beneflcial when used in the feed and matching networks for microwave and millimeter wave microstrip arrays. This is due to the fact that the maximum obtainable gain from an antenna array is limited by the ohmic losses in the metal microstrip transmission lines which form the feed network. Thus, even though the antenna directivity increases with an increasing number of elements, the gain is limited by the feed network losses. At mililmeter waves the problem becomes even more serious. However, recently Hansen ${ }^{7}$ showed an HTS 100 element Iinear array at $35 \mathrm{GHz}$ could experience a gain increase of 8 to $10 \mathrm{~dB}$ over an identical copper array.

NASA Lewis Research Center and Ball Aerospace have designed, fabricated and tested $30 \mathrm{GHz}$ four element superconducting microstrip arrays on various substrates. The antennas were assembled into a brass test fixture with a $50 \Omega$ gold microstrip feed line patterned on an alumina substrate separating the coax-to-microstrip transition (wiltron V-connector) from tho antenna (Fig. 4). Wire bonds connect the feed line to the antenna. Wire bonds were made directly to the YBCO. To test the devices, each test fixture was in turn mounted at the second stage of a 2-stage closed-cycle helium refrigerator. A hermetically sealed coaxial feedthrough passes the RF into the vacuum chamber. A high-density polyethylene (HDPE) cap serves as both a vacuum jacket and a radome. The entire cryrostat was mounted on the rotating pedestal of a far-field antenna range for pattern measurements in the recelve mode.

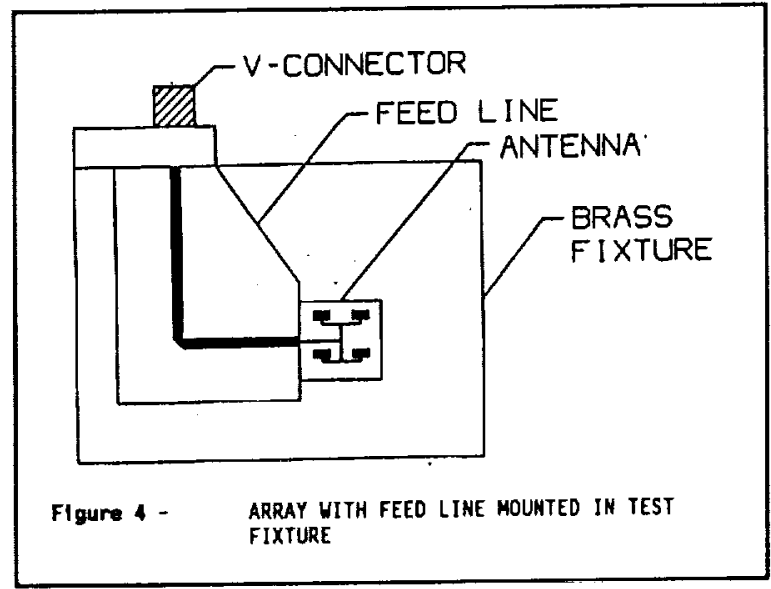

The measured $\mathrm{H}-\mathrm{plane}$ patterns are shown in Fig. 5 normalized to the highest recelved power for a magnesium oxide substrate. The antenna demonstrated a received power maxima at approximately $30.3 \mathrm{GHz}$ and 31.7 GHz. H-plane co- and cross-polarlzation measurements suggest that the lower erequency resonance is primarily due to feediline radiation. The amplitudes of the recelved antenna patterns at $70 \mathrm{~K}$ are comparable to gold levels. These results were anticipated for a first experiment because the HTS arrays are not yet fully optimized and the films used had a relatively low (below 85K) critical temperature $T_{c}$ ). Higher quality films and the use of ohmic contacts are expected to yield greater received powers for the HTS antennas relative to the gold circuits. It should be noted, though, that only $1 \mathrm{~dB}$ of improvement over the gold circuits can be expected for such a small number of 
elements.

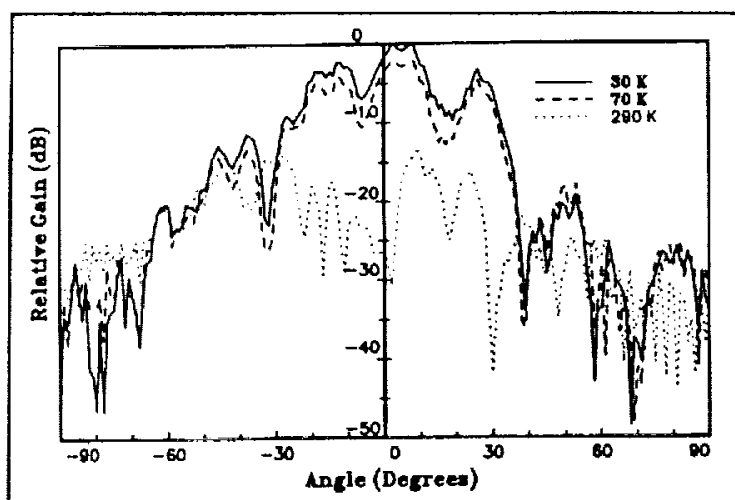

Figure 5 -

H-PIANE PATTERNS FOR YBaCUO ANTENKA OH MgO SUBSTRATE AT VARIOUS TEMPERATURES

The analytical and preliminary experimental work have shown that superconducting microstrip antennas have advantages over conventional antennas. It is also clear that the range of applications of superconducting antennas will be limited due to cryogenic requirements and the performance of superconducting films at frequencles above $50 \mathrm{GHz}$. However, in applications where high gain from the antenna is desired regardless of other considerations, high performance superconducting antennas will be likely candidates.

\section{Hicroveve supercenducter Bwitches}

The proposed superconductor switch consists of a rectangular patch of HTS material terminated by a quarter-wave radial stub, positioned one quarter wavelength away from a microstrip tee junction. If the switch is superconducting, its path appears as an ideal open circuit at the center frequency. It was determined that a 0.005 inch long, 25:1 aspect ratio HTS patch was nearly optimum for the design of a $32 \mathrm{GHz}$ switched ine phase shifter which will be described later.

Two possible modes of operation exist for a superconductor switch. The simplest is the bolometric mode, for which the film is held near its transition temperature. Normally, this technique exploits the fact that in this region, the temperature derivative of resistance is maximum. Incident radiation from an infrared light emitting diode or a laser raises the temperature and consequently causes the film to become resistive. This technique, although seemingly straightforward, has inherent speed limitations due to the high thermal conductivity of HTS compatible substrates. The problem for RF switching is compounded by the highly inductive component of the HTS near TC. It is apparent from the observation of HTS resonator $Q$ data that an acceptable reduction in loss for switching purposes doesn't occur until the temperature falls to about 758 of TC. Consequently, it is unlikely that a bolometric switch would be practical except under the most forgiving circumstances. To date, the fastest operation we achieved has been on the order of several seconds. Alternatively, magnetic field induced switching may provide a viable solution. The second operational mode is quantum mechanlcal. Incident photons with energy exceeding the bandgap break up the cooper pairs and generate a population of quasiparticles. This effect destroys the superconductivity, resulting in a resistive film. Speed is limited only by carrier recombination time. Implementation of this type of switch is hindered by the need to precisely control film stoichiometry, surface quality, and film thickness. Furthermore, although this effect has been well documented for low-Tc materials, there is still much controversy regarding an analogous response in high-Tc films.

In a typical millimeter-wave device requiring $R F$ switching, such as a phase shifter, the majority of insertion loss can be attributed to the parasitics associated with the switches. For example, metal line loss near $30 \mathrm{GHz}$ on 0.020 inch LaAlo is about $0.14 \mathrm{~dB}$ per wavelength. The parasitics of a typical MESFET switch could contribute as much as $0.5 \mathrm{~dB}$ to the total insertion loss. An "off" FET can be modeled as an RLC tank circuit. The inductor, $L$, is external to the FET and 18 included to resonate the circuit and Improve the off-state impedance. The capacitor, $c$, is the total pinch-off capacitance between the source and drain, and the resistor, $R$, is the channel and contact resistance. An "off" HTS switch can be modeled by an inductor and resistor. The inductor associated with the HTS switch represents the large kinetic inductance term of the superconductor near Tc. It is this reactive term which necessitates operating the HTS switch farther below the transition temperature than normal for a "bolometric" type of device. Both switches can be accurately modeled by a single resistor, Ron, in the high conductivity state. Sokolov defined a suitable figure of merit for FET switching devices as:

$$
Q^{2}=\left(R_{o n} R\right)^{-1}(w C)^{-2}=R_{o f f} / R_{o n}
$$

where $w$ is the radian frequency. For a typical switching FET, taking $R_{\text {on }}=10$ ohms, $C=0.07 \mathrm{pf}$, and $R=5$ ohms, $R_{\text {off }}$ is 1149 ohms at $30 \mathrm{GHz}$. This yields a figure of merit $\left(Q^{2}\right)$ of about 110 . If we consider an HTS switch, using a surface resistance of 0.010 ohms in the superconducting state, the equivalent resistivity at $30 \mathrm{GHz}$ is $8.4 \mathrm{e}-8$ ohm-cm. Assuming a normal state resistivity of $1 \mathrm{e}-3$ ohm-cm, by toggling between the two states, the theoretical ilgure of merit is about 12,000 . It is interesting to note that the semiconductor switching $\Omega$ will clegrade as the frequency 
squared; hence, it can be expected that an HTS switch will outperform a MESFET switch throughout the milimeter wavelength portion of the spectrum.

\section{An HT8 gwitched Iine phase ghifter}

Phased array antennas require variable phase shift components for each radiating element to achieve spatial scanning. A true time delay phase shifter can theoretically provide frequency independent beam steering for large arrays. A popular method for implementing this concept is referred to as the switched line technique. This method achieves true time delay by switching the signal between two alternate routes; elther the reference (zero phase shift) path or the delay path. The phase shift is proportional to the path length difference $(\Delta I)$ and frequency. Hence the time delay, which is equal to the derivative of phase with respect to radian frequency, will be $\Delta l / c$. The array scan angle will be frequency independent since the radiating element spacing and the delay have the same dependence on wavelength. A minimum of three active devices configured as two single-pole double-through switches are required per bit.

Several disadvantages are inherent to this method of implementation: asymmetric insertion loss between the delay and reference paths, high insertion loss overall, and poor isolation between the radio frequency (RF) and bias signals. The effect of switching $Q$ on insertion loss was discussed earlier. An equally vexing problem results from the interaction of the biasing network required to control the switches and the RF signal. standing wave patterns are set up on the bias lines causing unpredictable performance and sensitivity to bondwire lengths. The proposed phase shifter is shown in figure 6.

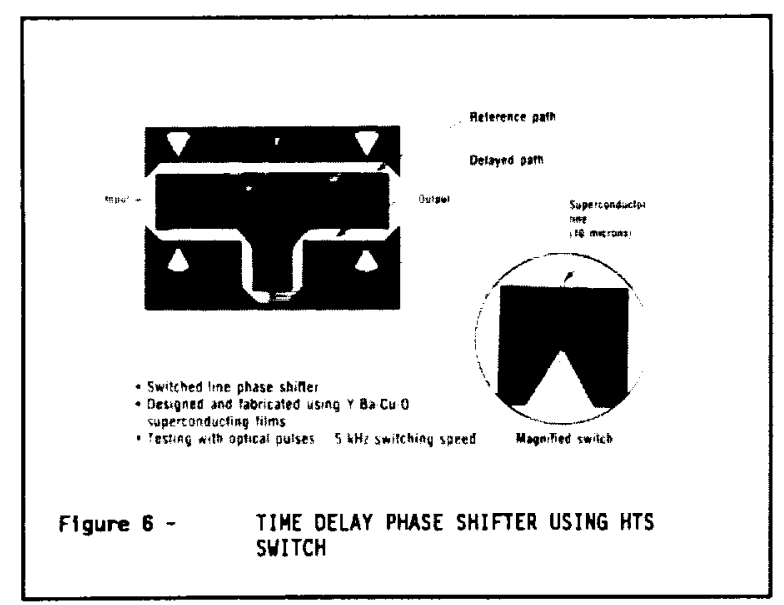

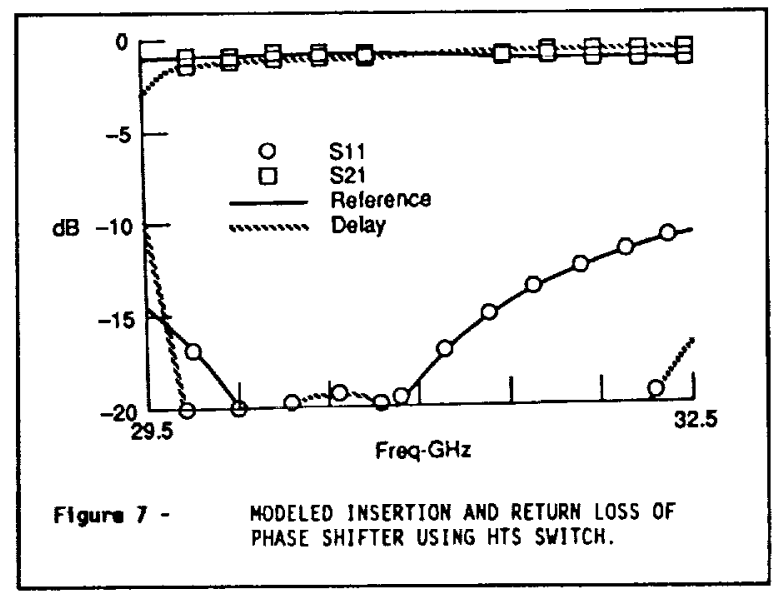

If the switches in the reference ine, for example, are superconducting they represent a high impedance path from the junction. concomitantly, the shunt switches in the delay path are caused to become normal (resistive) via optical illumination or magnetic quenching, passing the RF signal. The switches were modeled as thin film resistors of variable sheet resistance in the design, and the influence of the switch reactance was incorporated as part of the radial stub. Figure 7 illustrates the predicted performance and reveals the exceptionaliy narrow insertion loss envelope and low total insertion loss.

\section{Conclustons}

The potential for HTS devices and circuits to contribute significant performance enhancements to milimeter-wave communications subsystems has been demonstrated. Reproducible, large area films on attractive substrates will be enabling for practical applications, as will economical, compact, and reliable cryogenic cooling systems. It is expected that the combination of superconducting antennas, feed networks, filters, hybrid LNAs, and phase shifters would enormously enhance system performance in terms of the gain/temperature ratio.

\section{REFERENCES}

1. Martens, J. S., Ginley, D. S., Hietala, V. M., and Tigges, C. P., "Novel Applications of Ti-Ca-Ba-Cu-O Thin Films to Active and Passive High Frequency Devices, " Third International symposium on superconductivity, Sendal, Japan, Nov. 6-9, 1990.

2. Bhasin, K. B. et al, "Performance and Modeling of Superconducting Ring Resonators at Millimeter-Wave Frequencies," IEEE MTT-S International Microwave Symposium, Dallas, Tx. , May 8-10, 1990, pp. 269-272 (NASA TM102526). 
3. Swanson, A.W., "The Pseudomorphic HEMT," Microwaves and RF, March, 1987 , pp. $139-150$.

4. Duh, K.H.G. et al, "32-GHz Cryogenically Cooled HEMT LowNoise Amplifiers," IEEE Trans. Electron Devices, Vol. 36, No, 8, 1989, pp. 1528-1535.

5. Ponchak, D.S. et al. "A Technology Assessment of Alternative Communications systems for the space Exploration Initiative," AIAA space Programs and Technology Conference, Huntsville, Al., Sept. 25-27, 1990 (NASA TM-103243).

6. Dinger, R.J., "Some Potential Antenna Applications of High Temperature superconductors," Journal of superconductivity, vol. 3, No. 3, pp. 287-296, 1990 .

7. Hansen, R.C., "Superconducting Antennas," IEEE Trans. On Aerospace and Electronic Systems, vol. 26, No.2, pp. 345-354, March 1990.

8. Richard, M.A. et al, "Performance of a 4-Element $\mathrm{K}$-Band Thin Film Superconducting Microstrip Antenna," submitted to IEEE Microwave and Guided Wave Letters.

9. Sokolov, V. et al., "A Ka-Band GaAs Monolithic Phase Shifter," IEEE. Trans. Microwave Theory and Tech., MTT-31, No. 12,1983, pp. 1077-1082. 
Public reporting burden for this collectlon of Information is estlmated to average 1 hour per rosponse, including the time for revlewing instructions, searching oxisting data sources, gathering and maintaining the data needed, and completing and reviowing the collection of information. Send comments regarding this burden eatimate or any other aspect of this collection of information, including suggestions for reducing this burden, to Washington Headquarters Sorvices, Directorate for information Operations and Aeporta, 1215 Jefferson Davis Highway, Sulte 1204, Arlington, VA 22202-4302, and to the Office of Manapernent and Budget, Papenwork Reduction Project (0704-0188), Washington, DC 20503.

\begin{tabular}{|l|l|l}
\hline 1. AGENCY USE ONLY (Leave blank) & 2. REPORT DATE & $\begin{array}{r}\text { 3. REPORT TYPE AND DATES COVERED } \\
\text { Technical Memorandum }\end{array}$
\end{tabular}

4. TITLE AND SUBTITLE

High Temperature Superconductor Analog Electronics for

Millimeter-Wavelength Communications

6. AUTHOR(S)

R.R. Romanofsky and K.B. Bhasin
5. FUNDINO NUMBERS

WU 506-44-21
7. PERFORMING ORGANIZATION NAME(S) AND ADDRESS(ES)

National Aeronautics and Space Administration

Lewis Research Center

Cleveland, Ohio 44135 - 3191
8. PERFORMING ORGANIZATION

REPORT NUMBER

E- 6481
9. SPONSORINGMONITORING AGENCY NAMES(S) AND ADDRESS(ES)

National Aeronautics and Space Administration

Washington, D.C. $20546-0001$
10. SPONSORINOMONITORING AGENCY REPORT NUMBER

NASA TM- 105184

AIAA - 91-3592

11. SUPPLEMENTARY NOTES

Prepared for the Conference on Advanced Space Exploration Initiative Technologies cosponsored by the AIAA, NASA, and OAI, Cleveland, Ohio, September 4-6, 1991. Responsible person, R.R. Romanofsky, (216) 433-3507.

12a. DISTRIBUTIONAVAILABILITY STATEMENT

12b. DISTRIBUTION CODE

Unclassified - Unlimited

Subject Category 76

13. ABSTRACT (Maximum 200 words)

The performance of high-temperature superconductor (HTS) passive microwave circuits up to X-band has been encouraging when compared to their metallic counterparts. The extremely low surface resistance of HTS films up to about $10 \mathrm{GHz}$ enables a reduction in loss by as much as 100 times compared to copper when both materials are kept at about $77 \mathrm{~K}$. However, a superconductor's surface resistance varies in proportion to the frequency squared. Consequently, the potential benefit of HTS materials to millimeter-wave communications requires careful analysis. A simple ring resonator has been used to evaluate microstrip losses at Ka-band. We have also investigated additional promising components such as antennas and phase shifters. Prospects for HTS to favorably impact millimeter-wave communications systems are discussed.

14. SUBJECT TERMS

High temperature super conductors; Phased array antennas; Phase shift circuits

15. NUMBER OF PAQES

8

16. PRICE COOE

$\mathrm{AO} 2$

\begin{tabular}{|c|c|c|}
\hline $\begin{array}{c}\text { 17. SECURITY CLASSIFICATION } \\
\text { OF REPORT } \\
\text { Unclassified }\end{array}$ & $\begin{array}{c}\text { 18. SECURITY CLASSIFICATION } \\
\text { OF THIS PAGE } \\
\text { Unclassified }\end{array}$ & $\begin{array}{c}\text { 19. SECURITY CLASSIFICATION } \\
\text { OF ABSTAACT } \\
\text { Unclassified }\end{array}$ \\
\hline
\end{tabular}

NSN 7540-01-280-5500 
